\title{
Efecto de la temperatura de bulbo seco y humedad relativa en la sensación térmica percibida en espacios exteriores en clima cálido seco
}

\section{Effect of dry bulb temperature and relative humidity on the thermal sensation perceived in outdoors spaces in hot dry climate}

\author{
BOJÓRQUEZ-MORALES, Gonzalo†*, LUNA-LEÓN, Aníbal, ROMERO-MORENO Ramona y \\ JIMÉNEZ-LÓPEZ, Verónica
}

Universidad Autónoma de Baja California, Facultad de Arquitectura y Diseño, Programa de Maestría y Doctorado en Arquitectura, Urbanismo y Diseño, México.

ID $1^{\mathrm{er}}$ Autor: Gonzalo, Bojórquez-Morales / ORC ID: 0000-0001-9303-9278, Researcher ID Thomson: C-8687-2018, CVU CONACYT ID: 79645, SNI CONACYT ID: 45005

ID $1^{\text {er }}$ Coautor: Aníbal, Luna-León / ORC ID: 0000-0003-3480-0607, CVU CONACYT ID: 79641

ID $2^{\text {do }}$ Coautor: Ramona, Romero-Moreno / ORC ID: 0000-0002-5853-0229, Researcher ID Thomson: F-4992-2818, CVU CONACYT ID: 122232, SNI CONACYT ID: 33982

ID $3^{\text {er }}$ Coautor: Verónica, Jiménez-López / ORC ID: 0000-0003-1564-1479, CVU CONACYT ID: 399944

\section{Resumen}

Las condiciones del ambiente térmico en espacios exteriores determinan no solo la calidad del mismo, sino también el riesgo de morbilidad por permanecer en el. La presente investigación tuvo como objetivo estimar el efecto de la temperatura de bulbo seco y humedad relativa en la sensación térmica percibida en espacios exteriores en clima cálido seco. Se utilizó el enfoque de adaptación de confort térmico con el método de medias por intervalo de sensación térmica, el análisis consideró tres niveles de actividad para los periodos cálido (con 823 observaciones) frío (con 863 observaciones) y se estimó el nivel de adaptación de los sujetos en función de su sensación térmica. Los resultados indican que aun cuando el efecto critico es por la temperatura de bulbo seco, la humedad relativa tiene un efecto importante en la sensación percibida.

Sensación térmica percibida, Espacios exteriores, Clima cálido seco

\begin{abstract}
Conditions of the thermal environment in outdoor spaces determine not only its quality, but also the risk of morbidity from remaining in it. The objective of this research was to estimate the effect of dry bulb temperature and relative humidity on the perceived thermal sensation in outdoor spaces in hot dry climate. The thermal comfort adaptation approach was used with the thermal sensation interval means method, the analysis considered three activity levels for the warm period (with 823 observations) and cold period (with 863 observations), and the adaptation level of subjects based on their thermal sensation. The results indicate that even when the critical effect is due to the dry bulb temperature, the relative humidity has an important effect on the perceived sensation.
\end{abstract}

Perceived thermal sensation, Outdoor spaces, Hot dry climate

Citación: BOJÓRQUEZ-MORALES, Gonzalo, LUNA-LEÓN, Aníbal, ROMERO-MORENO Ramona y JIMÉNEZLÓPEZ, Verónica. Efecto de la temperatura de bulbo seco y humedad relativa en la sensación térmica percibida en espacios exteriores en clima cálido seco. Revista de Invención Técnica. 2020. 4-13:21-29.

*Correspondencia al Autor (Correo Electrónico: gonzalobojorquez@uabc.edu.mx)

$\dagger$ Investigador contribuyendo como primer autor. 


\section{Introducción}

Los riesgos de morbilidad y mortalidad por el uso de espacios públicos exteriores son una realidad en climas cálidos secos. Mexicali, Baja California presenta el mayor índice de mortalidad en México por golpes de calor (Díaz, Castro y Aranda (2013). Por otro lado, el conocer el efecto de la Temperatura de Bulbo Seco y la Humedad Relativa, sobre la sensación térmica percibida, permite visualizar condiciones de riesgo en el ambiente térmico urbano (CICC, 2009). El uso de un modelo de predicción por las implicaciones de variabilidad de ambientes térmicos y condiciones de los espacios exteriores y tiempos de permanencia no es adecuado, mientras que la aplicación del método de adaptación, debido a que es resultado de una evaluación de campo, se ajusta mejor a las condiciones exteriores (Höppe, 2002). La investigación sobre la sensación térmica percibida en exteriores es una necesidad para eventos como juegos olímpicos y ferias mundiales (Pickup y deDear, 2000), así como el uso de parques y espacios públicos exteriores (Nikolopoulou, 2004).

Hwang y Lin (2007), analizaron las condiciones de confort térmico en espacios exteriores y semi-exteriores, y demostraron como los sujetos disminuyen sus expectativas de confort térmico en espacios con esas características, por lo que aumentan su tolerancia a condiciones no confortables. Lin (2009) y Lin et al., (2011), desarrollaron un estudio sobre sensación y preferencia térmica en exteriores para periodo cálido y frío, en clima cálido húmedo. Se utilizó como modelo de referencia la nueva temperatura efectiva estándar (SET*). Los resultados demostraron la adaptación de los sujetos a los periodos estudiados y el efecto de las variables meteorológicas.

Bojórquez-Morales et al., (2012), realizaron un estudio de confort térmico en espacios exteriores, para tres niveles de actividad, en periodo cálido y periodo frío en un clima cálido seco extremoso. Las temperaturas neutrales fueron aproximadamente simétricas con respecto a sus rangos de confort térmico. Los sujetos en actividad intensa presentaron mejor adaptación a las condiciones climáticas. UríasBarrera (2019), desarrollo modelos de confort térmico para actividades deportivas intensas en espacios públicos exteriores.
Demostró que el nivel de actividad deportiva intensa amplia los rangos de neutralidad térmica, con valores que sobrepasan límites esperados por aclimatación.

La diferencia de este estudio con los mencionados es que se tuvo como objetivo estimar el efecto de la temperatura de bulbo seco y humedad relativa en la sensación térmica percibida en espacios exteriores en Mexicali, Baja California.

Se utilizó el enfoque de adaptación de confort térmico con el método de medias por intervalo de sensación térmica, el análisis consideró tres niveles de actividad para los periodos cálido (con 823 observaciones) frío (con 863 observaciones) y se estimó el nivel de adaptación de los sujetos en función de su sensación térmica.

Los resultados indican que aun cuando el efecto crítico es por la temperatura de bulbo seco, la humedad relativa tiene un efecto importante en la sensación percibida.

\section{Método}

El estudio de confort térmico en espacios exteriores, debido a la variabilidad de ambientes, condiciones de los espacios exteriores y tiempos de permanencia, no considera adecuado un modelo de predicción. Con base en lo anterior, se utilizó el enfoque de adaptación, con aplicación de encuestas con la escala de sensaciones percibidas de ISO 10551:2019, y uso simultáneo de un monitor de estrés térmico para registro de temperatura de bulbo seco (TBS) y humedad relativa (HR).

El trabajo de campo se realizó en el Centro Recreativo Juventud 2000 ubicado en Mexicali, Baja California (Latitud de 32'39'54" $\mathrm{N}$ y longitud de $115^{\circ} 27^{\prime} 21^{\prime \prime} \mathrm{O}, 4 \mathrm{~m} \mathrm{~s}$. n. m.). Clima es de tipo cálido seco extremoso, con temperaturas promedio de máximas de $42{ }^{\circ} \mathrm{C}$ (con máximas extremas de $49^{\circ} \mathrm{C}$ ) y temperaturas promedio de mínimas de $8{ }^{\circ} \mathrm{C}$, (con mínimas extremas de $-3{ }^{\circ} \mathrm{C}$ ) (Luna, 2008). 


\section{Instrumentos y variables}

La selección de variables a medir se basó en el efecto de las mismas en la sensación térmica percibida, para lo que se revisaron casos de estudio sobre confort térmico y medición en exteriores (del Campo y González, 2020; Oliveira y Andrade 2007; Hwang y Lin, 2007; Nikolopoulou, 2004; Spagnolo y deDear, 2003, Pickup y deDear, 2000; Potter y deDear, 2000 y Bojórquez-Morales et al., (2012)) y las normas ISO 7730(2005), ISO 7726(1998) e ISO 10551(2019).

Los instrumentos fueron seleccionados por precisión, rangos de medición y disponibilidad. Las variables seleccionadas fueron: temperatura de bulbo seco, humedad relativa, velocidad de viento y temperatura de globo gris (para este trabajo solo se presenta en análisis de TBS y HR). Se utilizó un monitor de estrés térmico que mide temperatura de bulbo seco, temperatura de bulbo húmedo, humedad relativa, velocidad de viento (omnidireccional) y temperatura de globo negro (Figura 1). El proceso realizado y los instrumentos cumplen con lo requerido en la norma ISO 7726 (1998), por lo que los datos generados son de Clase I, según la clasificación de Brager y deDear (1998). El cuestionario fue diseñado con base en la norma ISO 10551(2019) y en el análisis de tres cuestionarios para estudios de confort térmico (Nikolopoulou, 2004, Gómez-Azpeitia et al., 2007, Bojórquez-Morales et al., 2012).
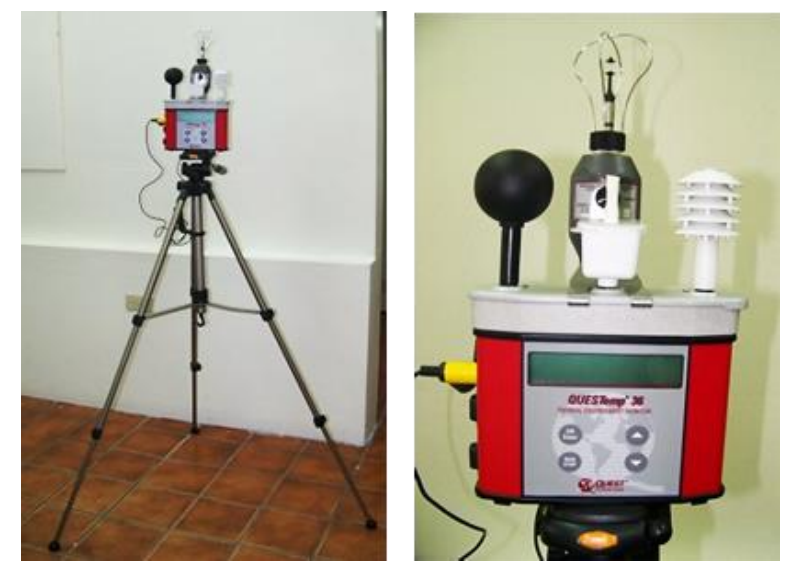

Figura 1 Monitor de estrés térmico Fuente: Tomada por los autores

\section{Trabajo de campo}

El diseño de la muestra se basó en el número de personas que asisten al parque recreativo de estudio en los periodos seleccionados. Se diseñó una muestra, con una confiabilidad del $95 \%$ y precisión de los estimadores del $5 \%$. El valor obtenido fue de 380 observaciones en promedio por periodo, debido a la aceptación del estudio entre los encuestados se llegó a 823 en periodo cálido y 863 en periodo frío.

Los instrumentos se revisaron antes de salir a campo y antes de iniciar la aplicación de las encuestas. La selección sujetos para la aplicación del cuestionario se planeó de forma aleatoria, pero debido a problemas de aceptación de los encuestados se procedió a hacerlo de forma determinística. Los sujetos de estudio fueron hombres y mujeres entre 12 y 65 años de edad, no se incluyeron individuos con condiciones biológicas irregulares como enfermedades temporales o crónicas, embarazo, periodo de lactancia o periodo menstrual. Las encuestas se realizaron en los horarios de operación del parque (06:00 a 22:00 horas de lunes a domingo), de tal forma que se cubrieron todos los periodos de uso en proporción al nivel de asistencia al lugar.

\section{Medias por intervalo de sensación térmica}

Se utilizó el método de medias por intervalo de sensación térmica (MIST) (Gómez-Azpeitia et al., 2007), que se basa en la propuesta de Nicol (1993) para climas “asimétricos” (Figura 2).

La diferencia fundamental del MIST con el método convencional es que antes de obtener la línea de regresión que caracteriza a la muestra estudiada, se determinan grupos o estratos de esta para calcular el valor promedio y la desviación estándar de cada uno de ellos.

Así, la regresión no se hace con todos los pares de datos de la muestra, sino sólo con los valores medios y los rangos se establecen mediante la adición y sustracción de una o dos veces la desviación estándar (DS) de la muestra. 


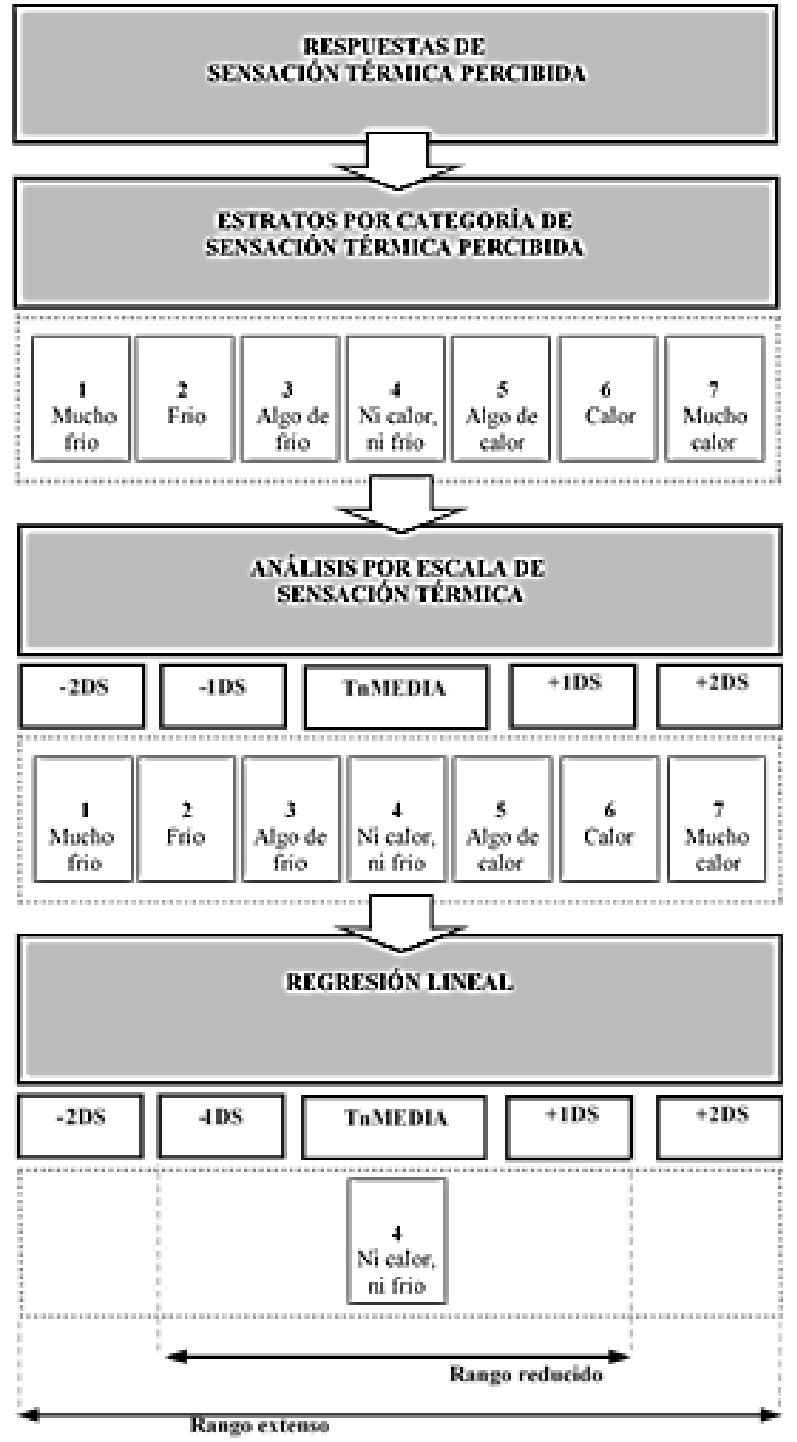

Nota: La representación de Temperatura neutral (Tn), cambia en función de la variable estudiada como Humedad neutral (Hn)

Figura 2 Temperatura neutral y rangos de confort térmico con el método de medias por intervalo de sensación térmica

Fuente: Bojórquez-Morales, 2010

El objetivo de este procedimiento es determinar el valor medio de temperatura de todas las repuestas de cada nivel de sensación térmica percibida. Con este procedimiento, se calcula el valor de la temperatura promedio de los sujetos que dijeron sentirse en confort térmico, pero también de quienes expresaron otras sensaciones térmicas. Con base en lo anterior, se procesaron por separado los datos colectados en el estudio de campo de acuerdo con cada una de las siete categorías de respuesta de confort térmico según ISO 10551 (2019).
Se determinaron para cada una de ellas los valores promedio y desviación estándar de las temperaturas registradas para cada respuesta colectada. Cuando el número de respuestas de determinado grupo no era suficiente para obtener resultados confiables, se omitió el procedimiento y se eliminó la categoría. La intersección de cada una de las líneas de regresión con la ordenada cuatro (que representa la sensación térmica de confort: ni calor, ni frío) determinan el valor de la temperatura neutral (Tn) o humedad neutral (Hn) según el método MIST, así como los valores límites de los rangos de confort térmico por TBS o HR.

El objetivo de este procedimiento es determinar el valor medio de temperatura de todas las repuestas de cada nivel de sensación térmica percibida. Con este procedimiento, se calcula el valor de la temperatura promedio de los sujetos que dijeron sentirse en confort térmico, pero también de quienes expresaron otras sensaciones térmicas. Con base en lo anterior, se procesaron por separado los datos colectados en el estudio de campo de acuerdo a cada una de las siete categorías de respuesta de confort térmico según ISO 10551 (2019).

\section{Análisis de la información}

El análisis de la información con respecto a la sensación térmica percibida fue de tipo mixto, cualitativo desde una perspectiva fenomenológica en inicio con base en las líneas de regresión, mediante el desarrollo de las premisas que se observaron con los resultados gráficos. Se hizo además un análisis cuantitativo con base en las características de la ecuación de regresión de la línea de regresión media, por temperatura de bulbo seco y humedad relativa.

\section{Sensación térmica percibida}

El estudio consideró por separado las observaciones para tres niveles de actividad, conforme a Fanger (1986) e ISO 8996, (2005): pasiva, modera e intensa. Los resultados presentados incluyen los tres niveles juntos. 


\section{Periodo cálido}

La sensación térmica por temperatura de bulbo seco mostró que los pocos casos que estuvieron fuera de los límites de confort térmico estimados, se presentaron hacia el extremo del rango extenso menor, además en lo que respecta al rango extenso mayor, no hubo casos que se aproximaran a esos valores. Lo anterior se debió a que en este caso se consideraron los tres niveles de actividad con diferentes características de adaptación térmica y psicológica de los sujetos, conforme a lo mencionado por Humphreys y Nicol, (2002) y Nikolopoulou y Steemers (2003) (Figura 3).

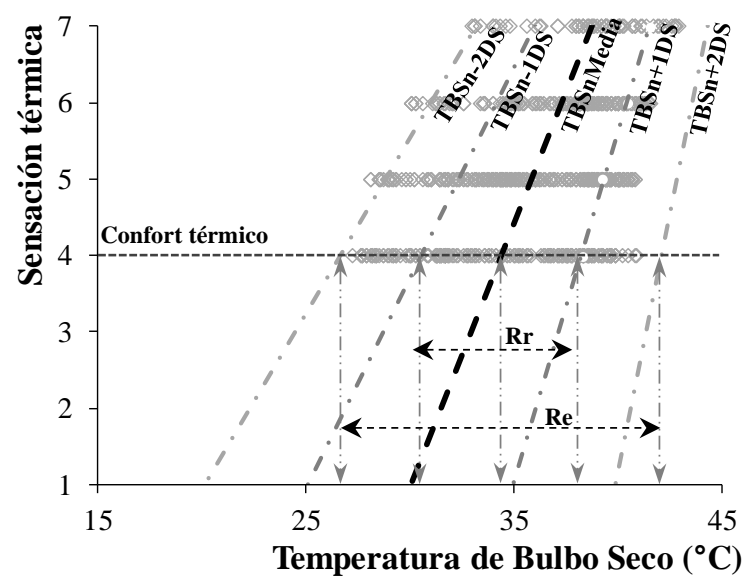

\begin{tabular}{|c|c|c|c|c|}
\hline TBSn-2DS & TBSn-1DS & $\mathbf{T B S n ~ M e d i a}$ & $\mathrm{TBSn}+1 \mathrm{DS}$ & $\mathrm{TBSn}+2 \mathrm{DS}$ \\
\hline $26.7^{\circ} \mathrm{C}$ & $30.5^{\circ} \mathrm{C}$ & $\mathbf{3 4 . 4}{ }^{\circ} \mathbf{C}$ & $38.2^{\circ} \mathrm{C}$ & $42.1^{\circ} \mathrm{C}$ \\
\hline
\end{tabular}

\begin{tabular}{|l|l|}
\hline Rango extenso (Re): $15.4^{\circ} \mathrm{C}$ & Rango Reducido $(\mathrm{Rr}): 7.7^{\circ} \mathrm{C}$ \\
\hline
\end{tabular}

Figura 3 Temperatura de Bulbo Seco neutral (TBSn) y rangos de confort térmico en periodo cálido

Fuente: Elaboración propia

Las líneas de regresión tuvieron una tendencia a ser convergentes con respecto a línea de regresión media, conforme se aumentó la sensación térmica de calor, lo que representó una mayor adaptación a las condiciones cálidas que a las frías, así como una variabilidad en las condiciones de adaptación térmica y psicológica de los sujetos, lo anterior se debió a que en este caso están incluidos los tres niveles de actividad. Con base en la desviación estándar, se puede decir que se presentaron diferencias significativas entre los niveles de adaptación para cada escala de sensación de los sujetos de estudio. Se observó una menor variabilidad conforme aumentó la temperatura, lo anterior indicó un mayor grado de adaptación a temperaturas altas en el periodo estudiado, lo que coincide con la teoría de adaptación de Humpreys y Nicol (2002) (Figura 3).
La sensación térmica por humedad relativa no presentó casos fuera de los límites de $\pm 2 \mathrm{DS}$; Sin embargo, se observaron algunos valores próximos al límite de -2DS. Lo anterior se debió a una mejor adaptación a las condiciones de humedad menores al valor de HRnMedia, que bajo condiciones cálidas y procesos de adaptación coincidieron con lo mencionado por Humphreys y Nicol (2002) y Nikolopoulou y Steemers (2003), además de lo mencionado por Urias-Barrera (2019); ya que a mayor humedad relativa aumentó la sensación de disconfort térmico (Figura 4).

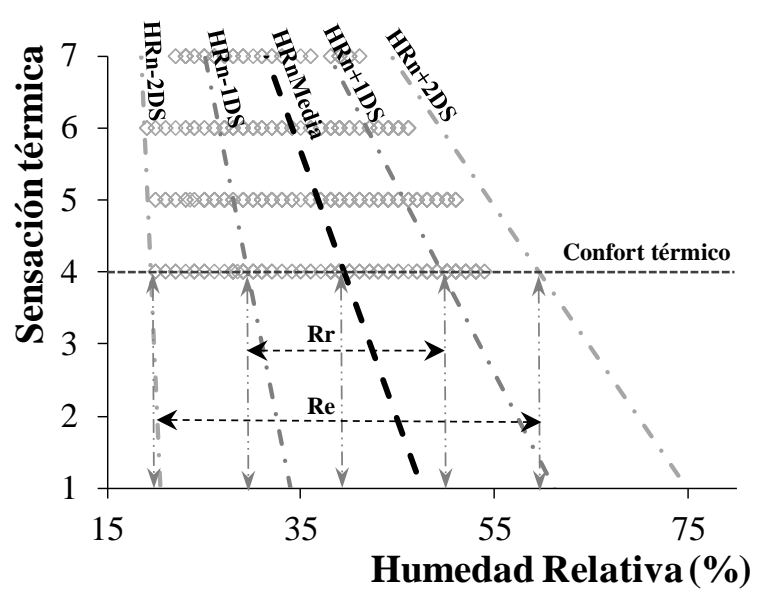

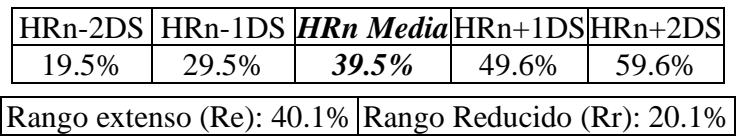

Figura 4 Humedad Relativa neutral (HRn) y rangos de confort térmico en periodo cálido

Fuente: Elaboración propia

Las líneas de regresión fueron convergentes con respecto a línea de regresión media, conforme se aumentó la sensación térmica de calor y se redujo la humedad relativa, lo que representó una mayor adaptación a las condiciones cálidas con menor humedad relativa.

Además de una variabilidad en las condiciones de adaptación térmica y psicológica de los sujetos, con base en las sensaciones térmicas percibidas de "ni calor, ni frío" hasta "mucho calor", lo anterior se debió a que en este caso están incluidos los tres niveles de actividad.

Con base en la desviación estándar se observaron diferencias entre los niveles de adaptación para las escalas de "ni calor, ni frío", "algo de calor" y "calor". 
Se presentó una menor variabilidad conforme se redujo la humedad relativa y aumentó la sensación térmica hacia "mucho calor", lo anterior indicó un mayor grado de adaptación a humedad relativa baja en el periodo estudiado, lo que coincidió con la teoría de adaptación de Humpreys y Nicol (2002) (Figura 4).

\section{Periodo frío}

La sensación térmica por temperatura de bulbo seco en periodo frío, mostró casos fuera de los límites de confort térmico hacia el extremo del rango extenso mayor (+2DS), lo que indicó menor grado de adaptación a temperaturas altas en este periodo. Lo cual se debió a que en este caso se consideraron los tres niveles de actividad con diferente actividad metabólica e intercambio energético con el ambiente térmico lo cual coincidió con Fanger (1972) e ISO 7730:2005 (Figura 5).

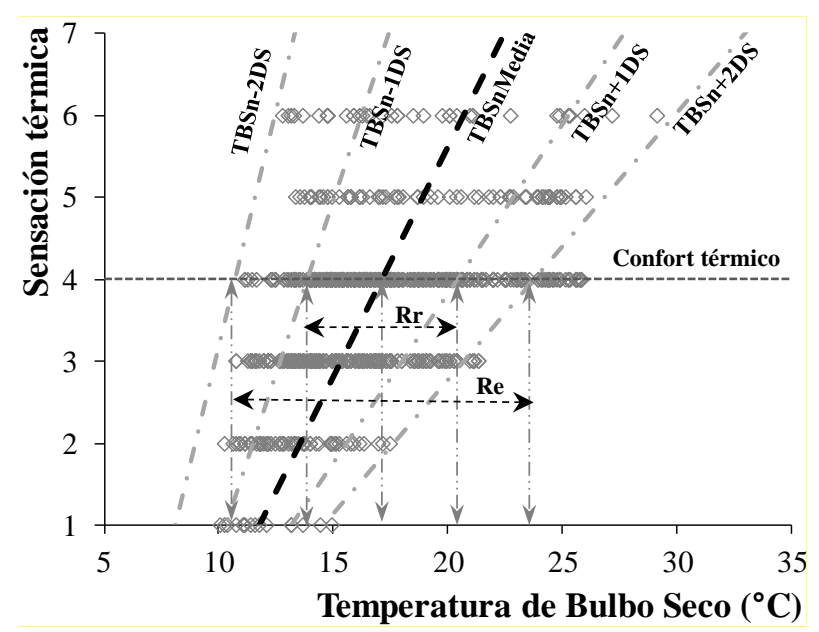

\begin{tabular}{|}
\begin{tabular}{|c|c|c|c|c|}
\hline TBSn-2DS & TBSn-1DS & TBSn Media & TBSn+1DS & TBSn+2DS \\
\hline $10.7^{\circ} \mathrm{C}$ & $13.9^{\circ} \mathrm{C}$ & $\mathbf{1 7 . 1}{ }^{\circ} \mathbf{C}$ & $20.4^{\circ} \mathrm{C}$ & $23.8^{\circ} \mathrm{C}$ \\
\hline
\end{tabular} \\
\hline
\end{tabular}

Figura 5 Temperatura de Bulbo Seco neutral (TBSn) y rangos de confort térmico en periodo frío

Fuente: Elaboración propia

Se observó una marcada reducción de la DS, conforme se redujo la sensación térmica a frío, con una convergencia significativa de las líneas de regresión con respecto a la línea de regresión media, lo que indicó una mayor adaptación a estas condiciones de ambiente térmico (frío) por parte de los sujetos estudiados, lo anterior es similar a lo afirmado por Höppe (2002) y Nikolopoulou y Steemers (2003).
Se presentó una reducción en la temperatura en la sensación térmica de "calor" con respecto a la de "algo de calor" lo cual se debió a los niveles de actividad metabólica de los sujetos en esos casos, esto es similar a lo establecido por Fanger (1972) (Figura 5).

La sensación térmica por humedad relativa en periodo frío mostró casos fuera de los límites de confort térmico, solo en la sensación térmica de "algo de frío" hacia el extremo del rango extenso mayor (+2DS), lo que indicó menor grado de adaptación a humedad relativa mayor a $67 \%$ en este periodo (Figura 6).

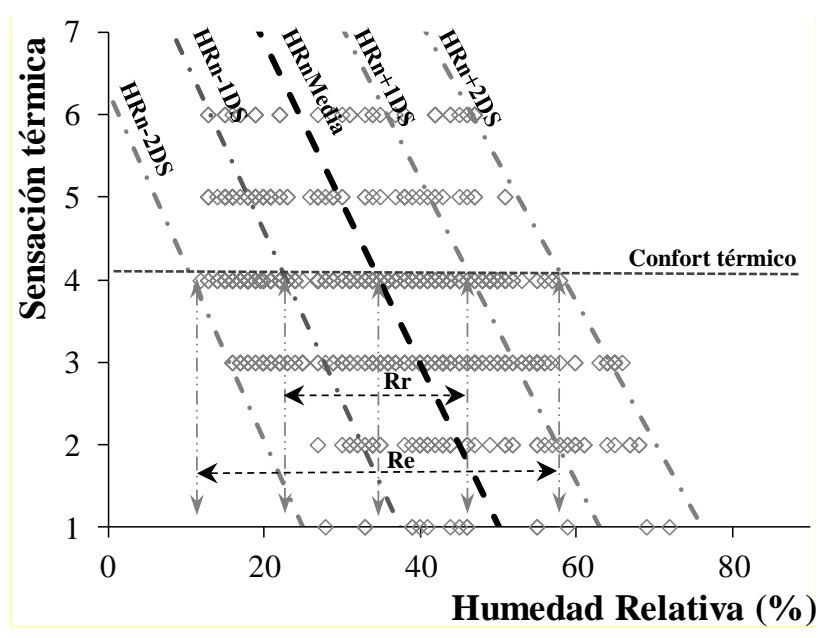

\begin{tabular}{|c|c|c|c|c|}
\hline HRn-2DS & HRn-1DS & HRn Media & HRn+1DS & HRn+2DS \\
\hline $10.8 \%$ & $22.8 \%$ & $\mathbf{3 4 . 7 \%}$ & $46.6 \%$ & $58.4 \%$ \\
\hline
\end{tabular}

\begin{tabular}{|l|l|}
\hline Rango extenso (Re): $47.6 \%$ & Rango Reducido (Rr): $23.8 \%$ \\
\hline
\end{tabular}

Figura 6 Humedad Relativa neutral (HRn) y rangos de confort térmico en periodo frío

Fuente: Elaboración propia

Es importante mencionar que se observó una inconsistencia en cuanto a la variación de DS de una escala de sensación térmica a otra, con cambios no mayores al $3 \%$ de humedad relativa lo cual no es significativo en la percepción del ambiente térmico conforme a lo establecido por Urias-Barrera (2019). Lo anterior se confirmó al observar que las líneas de regresión fueron visualmente paralelas entre sí, lo que indicó un nivel de adaptación similar en cada sensación térmica registrada. Además, se presentó una pendiente inclinada hacia los valores bajos de humedad relativa, lo que mostró una mejor adaptación a condiciones de poca humedad. Los rangos de confort obtenidos coincidieron con los valores propuestos por Urias-Barrera (2019), para exteriores e ISO 7730: 2005 para interiores (Figura 6). 


\section{Estudio comparativo}

El periodo cálido para la TBS (con base en su línea de regresión media) se caracterizó por tener una pendiente mayor que la HR en $46 \%$. En el caso del termino independiente de la TBS fue mayor que la HR en 5\%, aunque debido a la inclinación de las rectas el valor de TBS es negativo y el HR positivo. Con base en el coeficiente de determinación de la línea de regresión media de TBS, se observa una mayor representatividad comparada con HR, aun cuando el valor es del $1.5 \%$, denota una mayor influencia en el efecto de TBS (Tabla 1).

\begin{tabular}{|c|c|c|}
\hline $\begin{array}{c}\text { Variable } \\
\text { meteorológica }\end{array}$ & $\begin{array}{l}\text { Características } \\
\text { De la línea de } \\
\text { regresión media }\end{array}$ & $\begin{array}{c}\text { Valor } \\
\text { obtenido }\end{array}$ \\
\hline \multicolumn{3}{|l|}{ Periodo cálido } \\
\hline \multirow{3}{*}{$\begin{array}{l}\text { Temperatura } \\
\text { De bulbo } \\
\text { Seco }\end{array}$} & Pendiente de la recta & 0.6889 \\
\hline & Término & -19.689 \\
\hline & $\mathrm{R}^{2}(\mathrm{LRM})$ & 0.9982 \\
\hline \multirow{3}{*}{$\begin{array}{l}\text { Humedad } \\
\text { relativa }\end{array}$} & Pendiente de la recta & -0.3734 \\
\hline & Término & 18.757 \\
\hline & $\mathrm{R}^{2}(\mathrm{LRM})$ & 0.9833 \\
\hline \multicolumn{3}{|l|}{ Periodo frío } \\
\hline \multirow{3}{*}{$\begin{array}{l}\text { Temperatura } \\
\text { De bulbo } \\
\text { Seco }\end{array}$} & Pendiente de la recta & 0.5611 \\
\hline & Término & -5.6177 \\
\hline & $\mathrm{R}^{2}(\mathrm{LRM})$ & 0.9064 \\
\hline \multirow{3}{*}{$\begin{array}{l}\text { Humedad } \\
\text { relativa }\end{array}$} & Pendiente de la recta & -0.1961 \\
\hline & Término & 10.808 \\
\hline & $\mathrm{R}^{2}(\mathrm{LRM})$ & 0.8095 \\
\hline
\end{tabular}

Tabla 1 Características de la ecuación de regresión de la línea de regresión media, por temperatura de bulbo seco y humedad relativa

Fuente: Elaboración propia

El periodo frío presenta una pendiente de la recta $65 \%$ mayor para la TBS, con respecto a la HR. El termino independiente es mayor para HR en $48 \%$ con respecto a la TBS, y en este caso fue positivo para la HR y negativo para la TBS. Mientras que el coeficiente de determinación de la línea de regresión es $11 \%$ mayor para la TBS en comparación con la HR (Tabla 1).

\section{Conclusiones}

La temperatura de bulbo seco, presenta una variación significativa entre un periodo de estudio y otro. En los periodos cálido y frío, tiene condiciones extremas y características de clima asimétrico (según Nicol, 1993), mientras que en los periodos de transición se observan condiciones de un clima templado de tipo simétrico. El fenómeno de adaptación se observa claramente en los cambios de valor de la TBSn para cada periodo de estudio.
La humedad relativa, en Mexicali se mantiene todo el año entre 30 y $65 \%$, rango que está dentro de las condiciones de confort por HR establecidas por Cooper y Francis (1998). Lo anterior genera poca variación de porcentaje de HR entre cada escala de sensación térmica percibida, en los periodos estudiados y en todos los niveles de actividad. Esto ocasiona que los sujetos no desarrollen un proceso de adaptación a la humedad relativa ya que no hay cambios significativos de valores que estén fuera del rango entre confort y tolerables, por lo que en general el efecto de la humedad relativa es similar en la sensación térmica percibida por los sujetos durante los periodos estudiados.

Con base en la amplitud de rangos y trazo de las líneas de regresión para 1DS y 2DS, se puede afirmar que, en el periodo cálido, el efecto de la HR y TBS en la sensación térmica percibida, son similares ya que la variación de rangos es de 2 a 1 . Sin embargo, en el caso del periodo frío se observa que aun cuando se mantiene la relación de 2 a 1 en la amplitud de rangos, las líneas de regresión en la HR tienden a ser paralelas entre sí, mientras que en la TBS son convergentes hacia la sensación térmica de calor. Lo que indica una adaptación más uniforme con respecto a la HR, pero también demuestra el mayor efecto de la TBS.

En lo que respecta al efecto en la sensación térmica por temperatura de bulbo seco y humedad relativa, durante periodo frío se observa un mayor peso para la TBS en comparación con la HR, y aunque en el periodo cálido las diferencias son mínimas (1.5\%) el mayor peso también lo tiene la TBS.

Los resultados obtenidos pueden ser utilizados en procesos de diseño de espacios exteriores, como valores de referencia ante condiciones de riesgo por morbilidad en condiciones de ambiente térmico crítico, y en aplicaciones de indicadores de riesgo del efecto del clima sobre las condiciones humanas (UriasBarrera, 2019). 


\section{Agradecimientos}

A todo el personal de apoyo en la organización y desarrollo en el trabajo de campo. Al Centro recreativo Juventud 2000, Universidad Autónoma de Baja California y Universidad de Colima, por todo su apoyo. A los Doctores LucaBoJi y ZuliBoJi, por su tiempo prestado para la realización de este trabajo.

\section{Referencias}

Bojórquez-Morales G. (2010). Confort térmico en exteriores: actividades en espacios recreativos, en clima cálido seco extremo. Mimeo. Tesis de doctorado. Universidad de Colima.

Bojórquez-Morales G., Gómez-Azpeitia G., García-Cueto R., García-Gómez C., Luna-León A., Romero-Moreno R. (2012). Neutral temperature in outdoors for warm and cold periods for extreme warm dry climate. Proceedings of the 7th Windsor Conference: The Changing Context of Comfort in an Unpredictable World Cumberland Lodge, Windsor, UK, 12-15 April (2012). London: Network for Comfort and Energy Use in Buildings, http://nceub.org.uk

Brager, G. and Dear de, R. (1998). Thermal adaptation in the buil enviroment: a literature review. Energy and Buildings, 27, 83-96.

Comisión Intersecretarial de Cambio Climático (CICC), 2009, "Programa Especial de Cambio Climático 2009-2012", Diario Oficial de la Federación, México, D. F., 28 de agosto.

Cooper, C. y Francis C. (1998). People places: desing guidelines for urban open spaces. New York-Toronto: Jhon Wiley Sons, Inc.

Díaz, R., Castro, A., Aranda, P. (2013). Mortalidad por calor natural excesivo en el noroeste de México: Condicionantes sociales asociados a esta causa de muerte. Frontera norte vol.26 no.52 México jul./dic. 2014. ISSN 25940260 .

del Campo, N. U., y González, J. N. (2020). Bioclimática, mediciones ambientales y uso de los espacios urbanos: Evaluación comparativa en la Plaza de Chamberí, Madrid. REVISTARQUIS, 9(1), 1-26.
Fanger P.O. (1986). Thermal environmenthuman requirements. The environmentalist. Volume 6, Number 4, 275-278. Springer Netherlands.

Fanger, O. (1972). Thermal Comfort. New York: McGraw-Hill.

Gómez-Azpeitia, G., Ruiz, R., Bojórquez, G., Romero, R. (2007). Monitoreo de condiciones de confort térmico (Producto 3). (Comisión Nacional del Fondo para Vivienda. Proyecto Confort térmico y ahorro de energía en la vivienda económica en México, regiones de clima cálido seco y húmedo. CONAFOVI. 2004-01-20). Colima, Colima.

Höppe, P. (2002). Different aspects of assessing indoor and outdoor thermal comfort. Energy and Building, 34, 661-665.

Humphreys, M. and Nicol, F. (2002). The validity of ISO-PMV for predicting comfort votes in every-day thermal environments. Energy and Buildings, 34, 667-684.

Hwang, R.-L., Lin, T.-P. (2007). Thermal comfort requirements for occupants of semioutdoor and outdoor environments in hot-humid regions. Architectural Science Review, 50 (4), pp. 357-364.

Internacional Organization for Standardization. ISO 10551. (2019) Ergonomics of thermal enviroment - assessment of the influence of the thermal environment using subjective judgement scales. Ed. International Organization for Standarization, Ginebra, pp 52.

International Organization for Standardization. (2005). ISO 7730:2005 (E) Ergonomics of the thermal enviroment - analytical determination and interpretation of thermal comfort using calculation of the PMV and PPD indices and local thermal comfort criteria. Ginebra: Autor.

International Organization for Standarization. 7726, (1998). Ergonomics of the thermal enviroment - instruments for measuring physical quantities. Ginebra: International organizations for Standaritations. 
International Organization for Standardization. (2005). ISO 8996:2005 (E) Ergonomics of the thermal environment - Determination of metabolic heat production. Ginebra: Autor.

Lin, T.-P., deDear, R., Hwang, R.-1. (2011) Effect of thermal adaptation on seasonal outdoor thermal comfort. International Journal of Climatology, 31 (2), pp. 302-312.

Lin, T.-P. (2009) Thermal perception, adaptation and attendance in a public square in hot and humid regions. Building and Environment, 44 (10), pp. 2017-2026.

Luna, A. (2008). Diseño y evaluación de vivienda energéticamente sustentable. Tesis doctoral. Instituto de Ingeniería, Universidad Autónoma de Baja California, Mexicali.

Nicol, F. (1993) Thermal comfort "A handbook for field studies toward an adaptive model". London, University of East London.

Nikolopoulou, M. (2004). Designing open space in the urban environment: a bioclimatic approach. Attiki: Center for renewable energy sources.

Nikolopoulou, M., and Steemers, K. (2003). Thermal comfort and psychological adaptation as a guide for designing urban spaces. Energy and Buildings, 35, 95-101.

Oliveira, S., Andrade, H. (2007). An initial assessment of the bioclimatic comfort in an outdoor public space in Lisbon. International Journal of Biometeorology, 52 (1), pp. 69-84.

Pickup, J. and de Dear, R. (2000). An Outdoor Thermal Comfort Index (OUT_SET*) - Part I The Model and its Assumptions. In Biometeorology and Urban Climatology at the Turn of the Millennium. WCASP 50: WMO/TD No.1026. Edited by R.J. de Dear, J.D. Kalma, T.R.Oke and A. Auliciems. (WMO: Geneva). pp.279-283.

Potter, J. and de Dear, R. (2000). Field Study to Calibrate an Outdoor Thermal Comfort Index. In Biometeorology and Urban Climatology at the Turn of the Millennium. WCASP 50: WMO/TD No.1026. Edited by R.J. de Dear, J.D. Kalma, T.R.Oke and A.Auliciems. (WMO: Geneva). pp.315-320.
Spagnolo, J., deDear, R. (2003) A field study of thermal comfort in outdoor and semi-outdoor environments in subtropical Sydney Australia. Building and Environment, 38 (5), pp. 721-738.

Urias-Barrera H. (2019) Confort térmico en espacios públicos exteriores para actividades intensas deportivas: en clima cálido seco extremoso. Mimeo. Tesis de doctorado. Universidad Autónoma de Baja California 